\title{
Heavy Particle Clustering in Turbulent Flows
}

\author{
Jérémie Bec ${ }^{1}$, Luca Biferale ${ }^{2}$, Massimo Cencini ${ }^{3}$, Alessandra Lanotte ${ }^{4}$,
} Stefano Musacchio ${ }^{5}$, and Federico Toschi ${ }^{6}$

1 CNRS UMR6202, Observatoire de la Côte d'Azur, BP4229, 06304 Nice Cedex 4, France. jeremie.bec@obs-nice.fr

2 Dept. of Physics and INFN, University of Rome "Tor Vergata", Via della Ricerca Scientifica 1, 00133 Roma, Italy.

3 INFM-CNR, SMC Dept. of Physics, University of Rome "La Sapienza", Piazzale A. Moro 2, 00185 Roma, Italy.

4 CNR-ISAC and INFN, Sezione di Lecce, Str. Prov. Lecce-Monteroni, 73100 Lecce, Italy.

${ }^{5}$ INLN, 1361 Route des Lucioles, 06560 Valbonne, France.

${ }^{6}$ CNR-IAC, Viale del Policlinico 137, 00161 Roma, Italy, and INFN, Sezione di Ferrara, Via G. Saragat 1, 44100 Ferrara, Italy.

\begin{abstract}
Distributions of heavy particles suspended in incompressible turbulent flows are investigated by means of high-resolution direct numerical simulations. It is shown that particles form fractal clusters in the dissipative range, with properties independent of the Reynolds number. Conversely, in the inertial range, the particle distribution is not scale-invariant. It is however shown that deviations from uniformity depends only on a rescaled contraction rate, and not on the local Stokes number given by dimensional analysis. Particle distribution is characterized by voids spanning all scales of the turbulent flow; their signature on the coarse-grained mass probability distribution is an algebraic behavior at small densities.
\end{abstract}

Keywords: particle-laden flows, preferential concentration, inertial particles, turbulent transport

\section{Introduction}

Spatial disributions of finite-size heavy impurities suspended in incompressible flows is a crucial issue in engineering [1], planetology [2] and cloud physics [3]. Such particles possess inertia, and generally distribute in a strongly inhomogeneous manner. The common understanding of such preferential concentrations relies on the idea that, in a turbulent flow, vortices act as centrifuges ejecting such heavy particles [4]. This picture was successfully used to describe the small-scale particle distribution and, in particular, to show that it depends only on the Stokes number $\mathcal{S}_{\eta}=\tau / \tau_{\eta}$ which is obtained by nondimensionalizing the particle response time $\tau$ with the characteristic time $\tau_{\eta}$ of the small turbulent eddies.

Y. Kaneda (ed.), IUTAM Symposium on Computational Physics and New Perspectives in Turbulence, 79-84.

(C) 2008 Springer. 
We confirm here that such a description is relevant at length scales which are smaller than the dissipative scale $\eta$ of the fluid turbulent flow. In particular, maximal clustering is found for Stokes numbers of the order of unity. However, we show that particle concentration experiences also very strong fluctuations at scales within the inertial range of turbulence. In analogy with small-scale clustering, it is expected that for $r \gg \eta$ the relevant parameter is the local Stokes number $\mathcal{S}_{r}=\tau / \tau_{r}$, where $\tau_{r}$ is the characteristic time of eddies of size $r$ [5]. Surprisingly, we present evidences which are reported with more details in [6], that such a dimensional argument does not apply to describe the organization of particles in the inertial range of turbulence.

\section{Model and DNS}

In very dilute suspensions, small particles which are much heavier than the fluid evolve according to the Newton equation [7]

$$
\tau \ddot{\boldsymbol{X}}=\boldsymbol{u}(\boldsymbol{X}, t)-\dot{\boldsymbol{X}}
$$

where buoyancy is neglected. The response time $\tau$ is proportional to the square of the particles size and to their density contrast with the fluid. The fluid velocity $\boldsymbol{u}$ is a given solution to the incompressible Navier-Stokes equation obtained numerically by direct pseudo-spectral simulations on cubic grids of size $\mathcal{L}=2 \pi$ with $128^{3}, 256^{3}$ and $512^{3}$ collocation points corresponding to Taylor micro-scale Reynolds numbers $R e_{\lambda} \approx 65,105$ and 185, respectively. The fluid flow is forced by keeping constant the energy content in the two first shells in Fourier space. Particles $(N=7.5$ millions for each set of 15 different Stokes number in the range $\left.\mathcal{S}_{\eta} \in[0.16: 3.5]\right)$, initially seeded homogeneously in space with velocities equal to the local fluid velocity, are evolved with (1) for about two large-scale eddy turnover times. After this time, a statistical steady state is reached and measurements are performed. Details on the numerics are reported in [8].

\section{Small-Scale Clustering}

Below the Kolmogorov scale $\eta$, the velocity field is differentiable and the motion of particles is governed by the fluid strain. Their dissipative dynamics leads their trajectories to converge to a dynamically evolving attractor, so that their mass distribution is singular and generically scale-invariant with fractal properties at small scales. In order to characterize such particle clusters we measured the correlation dimension $\mathcal{D}_{2}$, which is estimated through the small-scale behavior of the probability to find two particles at a distance less than a given $r: P_{2}(r) \sim r^{\mathcal{D}_{2}}$. The dependence of $\mathcal{D}_{2}$ on $\mathcal{S}_{\eta}$ and $R e_{\lambda}$ is shown in Fig. 1 (Left). We first notice that $\mathcal{D}_{2}$ depends very weakly on $R e_{\lambda}$, at least in the range of Reynolds numbers explored here. This observation agrees with a recent numerical study [9], where particle clustering was equivalently characterized in terms of the radial distribution function. This confirms 

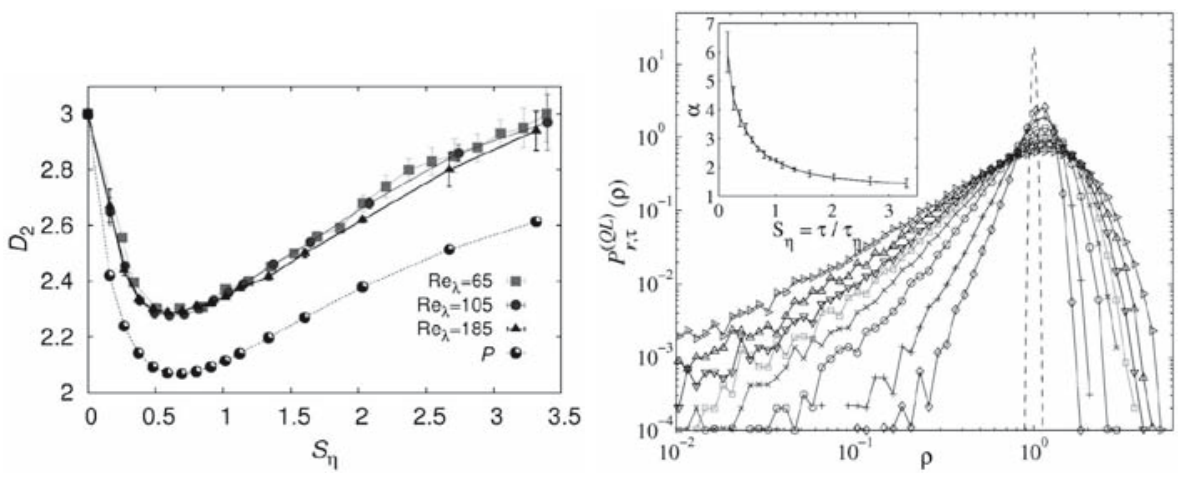

Fig. 1. Left: Correlation dimension $\mathcal{D}_{2}$ vs $\mathcal{S}_{\eta}$ for three different $R e_{\lambda}$, and probability $\mathcal{P}$ to find particles in non-hyperbolic (rotating) regions of the flow, shown for $R e_{\lambda} \approx 185$ (multiplied by an arbitrary factor for plotting purposes). Right: QuasiLagrangian PDF of the coarse-grained mass density $\rho_{r}$ in log-log scale for $\mathcal{S}_{\eta}=0.27$, $0.37,0.58,0.80,1.0,1.33,2.03,3.31$ (from bottom to top) at scale $r=32$ grid points and for $R e_{\lambda} \approx 185$. The solid line represent the Poisson distribution. Inset: exponent $\alpha$ of the left algebraic tail vs $\mathcal{S}_{\eta}$.

that $\tau_{\eta}$, which varies by more than a factor 2 between the smallest and the largest Reynolds number considered here, is the only relevant time scale to characterize clustering below $\eta$ and in particular that the effects of the flow intermittency cannot be detected. For all values of $R e_{\lambda}$, a maximum of clustering (corresponding to a minimum of $\mathcal{D}_{2}$ ) is observed for $\mathcal{S}_{\eta} \approx 0.6$. Particle positions strongly correlate with the local structure of the fluid velocity field. This is evidenced in Fig. 1 where is plotted the probability $\mathcal{P}$ to find particles in non-hyperbolic regions of the flow, i.e. at those points where the strain matrix has two complex conjugate eigenvalues. This is consistent with the traditional view relating particle clustering to vortex ejection.

\section{Inertial Range Clustering}

Fluctuations in the particle spatial distribution are qualitatively observed to extend far inside the inertial range. To quantify this effect, we consider the probability density function $(\mathrm{PDF}) P_{r, \tau}^{(Q L)}(\rho)$ of the quasi-Lagrangian particle density coarse-grained on a scale $r$ inside the inertial range. This quantity is obtained by looking at the mass distribution in balls centered on a given particle trajectory. This amounts to weighting each cell with the mass it contains; for statistically homogeneous distributions, the quasi-Lagrangian density is related to the Eulerian density by $\left\langle\rho_{r}^{p}\right\rangle_{Q L}=\left\langle\rho_{r}^{p+1}\right\rangle_{E u l}$ (see [10] for more details and a precise definition of quasi-Lagrangian averages). For tracers, which are uniformly distributed, this PDF tends for infinite number of particles, $N \rightarrow \infty$, to a delta function centered at $\rho=1$. For a large but finite $N$, it is given by the asymptotic behavior of the binomial distribution. 
As seen from Fig. 1 (Right), strong deviations from a uniform distribution can clearly be measured at both moderately small response times and for length scales inside the inertial range. This indicates that concentration fluctuations are important not only at dissipative scales but also in the inertial range. A noticeable observation is that the low-density tail of the PDF (related to voids) displays an algebraic behavior $P_{r, \tau}^{(Q L)}(\rho) \sim \rho^{\alpha(r, \tau)}$. The dependence of such an exponent for fixed $r$ and varying the Stokes number $\mathcal{S}_{\eta}$ is shown in the inset of Fig. 1 (Right). For low inertia $\left(\mathcal{S}_{\eta} \rightarrow 0\right)$ the exponent tends to infinity in order to recover the non-algebraic behavior of tracers. At larger Stokes numbers the exponent approaches $\alpha=1$, indicating a non-zero probability for totally empty areas.

Fixing the response time $\tau$ and increasing the observation scale $r$ reproduces the same qualitative picture as fixing $r$ and decreasing $\tau$. A uniform distribution is recovered in both limits $r \rightarrow \infty$ or $\tau \rightarrow 0$. These two limits are actually equivalent. At length-scales $r \gg \eta$ within the inertial range, the fluid velocity field is not smooth: according to Kolmogorov (K41) theory, velocity increments behave as $\delta_{r} u \sim(\varepsilon r)^{1 / 3}$. Standard turbulence theory suggests that the physics at scale $r$ is associated to time scales of the order of the turnover time $\tau_{r}=r / \delta_{r} u \sim \varepsilon^{-1 / 3} r^{2 / 3}$. It is then clear that, for any finite particle response time $\tau$, the local inertia measured by $\mathcal{S}_{r}=\tau / \tau_{r}$ becomes so small at sufficiently large scales that particles should behave as tracers and so distribute uniformly in space [5]. Deviations from uniformity for finite $\mathcal{S}_{r}$ are expected not to be scale-invariant [11]. In particular, phenomenology suggests that the particle distribution should depend only on the local Stokes number $\mathcal{S}_{r}$ as observed in random $\delta$-correlated in time flows [12]. However this argument does not take into consideration some important aspects of realistic flows.

We first notice that (1) can be rewritten as $\boldsymbol{V}=\boldsymbol{u}-\tau \boldsymbol{A}$, where $\boldsymbol{V}=\dot{\boldsymbol{X}}$ denotes the velocity of the particle and $\boldsymbol{A}=\ddot{\boldsymbol{X}}$ its acceleration. Far enough in the inertial range, we have $\mathcal{S}_{r} \ll 1$ and the particle acceleration can be approximated with the fluid acceleration: $\boldsymbol{A} \approx \boldsymbol{a}=\partial_{t} \boldsymbol{u}+\boldsymbol{u} \cdot \nabla \boldsymbol{u}$. As a consequence, the particles evolve as if transported by a synthetic compressible flow $[7,11]$ whose divergence is $\boldsymbol{\nabla} \cdot \boldsymbol{V}=-\tau \nabla \cdot(\boldsymbol{u} \cdot \boldsymbol{\nabla} \boldsymbol{u}) \approx \tau \nabla^{2} p$. In this effective velocity field, the only time scale $\mathcal{T}_{r, \tau}$ relevant to the distribution of particles at scale $r$ is given by the inverse of the contraction rate of a volume of size $r$, i.e.

$$
1 / \mathcal{T}_{r, \tau}=\left(1 / r^{3}\right) \int_{[0, r]^{3}} \nabla \cdot \boldsymbol{V} d^{3} x \approx\left(1 / r^{3}\right) \int_{[0, r]^{3}} \tau \nabla^{2} p d^{3} x .
$$

Figure 2 (Left) displays the power spectrum of the pressure $E_{p}(k)$, pressure gradients $E_{\nabla p}(k)$ and of the velocity field $E_{u}(k)$. As one can see in the range of wavenumbers where the $\mathrm{K} 41$ scaling is observed, i.e. where $E_{u}(k) \sim k^{-5 / 3}$, we find that $E_{p}(k) \sim k^{-5 / 3}$ and $E_{\nabla p}(k) \sim k^{1 / 3}$, suggesting that $\delta_{r} p \sim r^{1 / 3}$ and $\delta_{r} \nabla p \sim r^{-2 / 3}$, so that the scaling of pressure is there dominated by sweeping, i.e. $\delta_{r} \nabla p=\nabla \delta_{r} u^{2} \sim U(\varepsilon r)^{1 / 3} / r$, where $U$ is the root mean square velocity. 

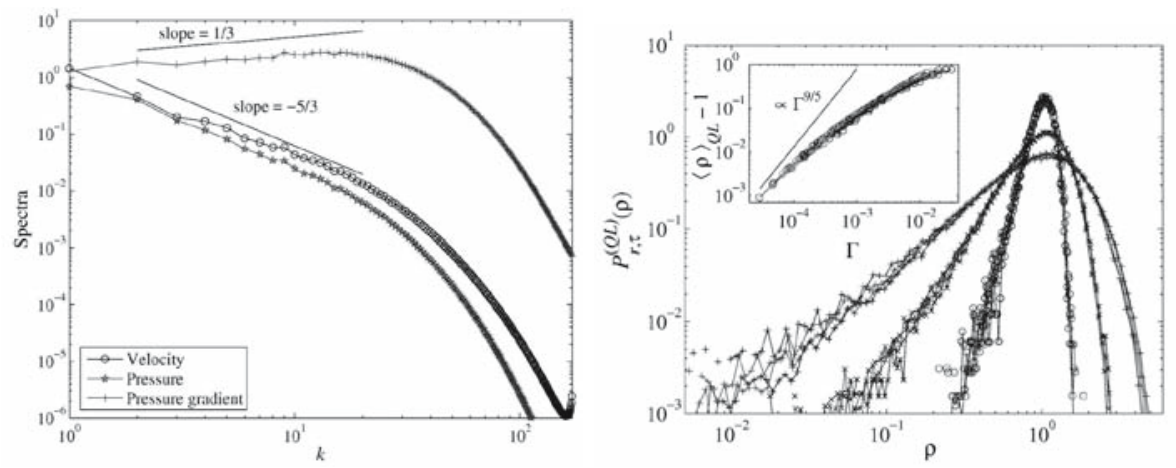

Fig. 2. Left: Pressure, pressure gradient and energy spectra vs the wavenumber $k$. The straight lines indicate the scaling $k^{-5 / 3}$ and $k^{1 / 3}$. Right: PDF of the coarsegrained mass in the inertial range for three values of the non-dimensional contraction rate $\Gamma$ (different collapsing curves refer to different Stokes numbers and different scales). From bottom to top: $\Gamma=4.810^{-4}, \Gamma=2.110^{-3}$, and $\Gamma=7.910^{-3}$ (for $\left.\mathcal{S}_{\eta}=1.60,2.03,2.67,3.31\right)$. Inset: deviation from unity $\langle\rho\rangle_{Q L}-1$ of the first-order QL moment for scales $r$ within the inertial range. For comparison, the behavior $\propto \Gamma^{9 / 5}$ obtained when assuming point clusters of particles is shown as a solid line. Both figures refer to $R e_{\lambda} \approx 185$.

Note that the scaling we observe is likely to be due to finite Reynolds number effects [13]. After one integration by part of (2), and plugging the above scaling, the integral can be dimensionally estimated as $1 / \mathcal{T}_{r, \tau} \sim \tau U \varepsilon^{1 / 3} r^{-5 / 3}$. We thus expect that the PDF of the coarse-grained mass density is not a function of $r$ and $\tau$ separately, but of the dimensionless contraction rate $\Gamma=\tau \lambda \varepsilon^{1 / 3} r^{-5 / 3}$, where we have adimensionalized $\mathcal{T}_{r, \tau}$ with the reference time $\lambda / U$.

Figure 2 (Right) shows $P_{r, \tau}^{(Q L)}(\rho)$ for three choices of $\Gamma$ obtained from different sets of values of $r$ and $\tau$. The different curves collapse, giving strong evidence in favor of the above argument. In particular, as represented in the inset of Fig. 2 (Right), the deviations from unity of the first moment of density collapse for all $\mathcal{S}_{\eta}$ investigated and for all scales inside the inertial range of our simulation. This quantity is the same as the Eulerian 2nd-order moment and gives the probability $P_{2}(r)$ to have two particles within a distance $r$ (here inside the inertial range). The particle distribution recovers uniformity at large scales very slowly: much slower than if they were distributed as Poisson point-like clusters, for which $\left\langle\rho^{2}\right\rangle-1 \propto r^{-3} \propto \Gamma^{9 / 5}$ (shown in the inset for comparison).

\section{Summary and Conclusions}

In conclusions we have shown that fluctuations of the concentration of heavy particles are important, not only in the dissipative range of turbulence where 
they can be described by using tools borrowed by dynamical systems theory, but also in the inertial range. Moreover, the PDF of the coarse-grained mass density displays a rescaling property in the inertial range and only depends on $\Gamma=\tau \lambda \varepsilon^{1 / 3} r^{-5 / 3}$, in relation with the scaling properties of the pressure field. The presence of intermittency corrections cannot be excluded for high-order statistics but they are not detectable by the present investigation. These findings may be important for developing models for water droplet growth and the scavenging of aerosol particles. Worth of further investigation is a characterization of the large fluctuations of the particle density field by means of statistical cluster analysis tools, such as minimum spanning trees or Minkovski functionals.

We benefited from useful discussions with G. Boffetta, A. Celani and A. Fouxon. This work has been partially supported by the EU under contract HPRNCT-2002-00300 and the Galileo program on particle suspensions. DNS were performed at CINECA (Italy) and IDRIS (France) under the HPC-Europa programme (RII3-CT-2003-506079). The unprocessed data of this study are freely available from http://cfd.cineca.it.

\section{References}

1. Crowe C, Sommerfeld M, Tsuji Y (1998) Multiphase Flows with Particles and Droplets. CRC Press, New York

2. de Pater I, Lissauer J (2001) Planetary Science. Cambridge University Press, Cambridge

3. Pruppacher H, Klett J (1996) Microphysics of Clouds and Precipitation, Kluwer Academic Publishers, Dordrecht

4. Fessler J, Kulick J, Eaton J (1994) Phys Fluids 6:3742-3749

5. Falkovich G, Fouxon A, Stepanov M (2003) Statistics of turbulence-induced fluctuations of particle concentration. In Gyr A, Kinzelbach W (eds) Sedimentation and Sediment Transport. Kluwer Academic Publishers, Dordrecht

6. Bec J, Biferale L, Cencini M, Lanotte A, Musacchio S, Toschi F (2006) Preprint nlin.CD/0608045

7. Maxey M (1987) J Fluid Mech 174:441-465

8. Bec J, Biferale L, Boffetta G, Celani A, Cencini M, Lanotte A, Musacchio S, Toschi F (2006) J Fluid Mech 550:349-358

9. Collins L, Keswani A (2004) New J Phys 6:119

10. Bec J, Gawȩdzki K, Horvai P (2004) Phys Rev Lett 92:224501

11. Balkovsky E, Falkovich G, Fouxon A (2001) Phys Rev Lett 86:2790-2793

12. Bec J, Cencini M, Hillerbrand R (2006) Phys Rev E in press nlin.CD/0606038

13. Gotoh T, Fukayama D (2001) Phys Rev Lett 86:3775-3779 